\title{
High Shear Wet Milling Mixing
}

National Cancer Institute

\section{Source}

National Cancer Institute. High Shear Wet Milling Mixing. NCI Thesaurus. Code C113028.

Mixing of solids into liquid or of immiscible liquids by shear and impact within high velocity streams. 